Review Article

\title{
Genetic Determinants of Resistance among ESBL-Producing Enterobacteriaceae in Community and Hospital Settings in East, Central, and Southern Africa: A Systematic Review and Meta-Analysis of Prevalence
}

\author{
Onduru G. Onduru $\mathbb{D}^{1},{ }^{1}$ Rajhab S. Mkakosya, ${ }^{1}$ Said Aboud, ${ }^{2}$ and Susan F. Rumisha ${ }^{3}$ \\ ${ }^{1}$ Department of Microbiology, College of Medicine, University of Malawi, Private Bag 360, Zomba, Malawi \\ ${ }^{2}$ Department of Microbiology and Immunology, Muhimbili University of Health and Allied Sciences, P.O. Box 65001, \\ Dar es Salaam, Tanzania \\ ${ }^{3}$ Directorate of Information Technology and Communication, National Institute for Medical Research, P.O. Box 9653, \\ Dar es Salaam, Tanzania \\ Correspondence should be addressed to Onduru G. Onduru; ogyonduru@yahoo.com
}

Received 13 May 2020; Revised 17 August 2020; Accepted 29 May 2021; Published 3 June 2021

Academic Editor: José A. Oteo

Copyright (C) 2021 Onduru G. Onduru et al. This is an open access article distributed under the Creative Commons Attribution License, which permits unrestricted use, distribution, and reproduction in any medium, provided the original work is properly cited.

\begin{abstract}
Background. The world prevalence of community and hospital-acquired extended-spectrum $\beta$-lactamase (ESBL)-producing Enterobacteriaceae is increasing tremendously. Bacteria harboring ESBLs are currently the number one critical pathogens posing a major threat to human health. Objective. To provide a summary of molecular evidence on the prevalence of ESBL-producing Enterobacteriaceae (ESBL-E) and associated genes at community and hospital settings in East, Central, and Southern African countries. Methods. We conducted a systematic literature search on PubMed and Google Scholar databases for the available molecular studies on ESBL-E in hospitals and community settings in East, Central, and Sothern Africa (ECSA). Published studies in English language involving gene characterization of ESBLs from human samples in hospital and community settings were included in the review, inception to November 2019. A random effect meta-analysis was performed to estimate the prevalence of ESBL-E. Results. A total of 27 studies involving molecular characterization of resistance genes from 20,225 ESBL-E isolates were included in the analysis. Seventy-four percent of all studies were hospital based, $15 \%$ were based in community settings, and others were done in both hospital and community settings. Of all the studies, $63 \%$ reported E. coli as the dominant isolate among ESBL-E recovered from clinical samples and Klebsiella pneumoniae was reported dominant isolates in 33\% of all studies. A random pooled prevalence of ESBL-E was 38\% (95\% CI $=24-53 \%)$, highest in Congo, 92\% (95\% CI =90-94\%), and lowest in Zimbabwe, 14\% (95\% CI $=9-20 \%)$. Prevalence was higher in hospital settings $41 \%$ (95\% CI $=23-58 \%$ ) compared to community settings 34\% (95\% $\mathrm{CI}=8-60 \%)$. ESBL genes detected from clinical isolates with ESBL-E phenotypes in ECSA were those of Ambler molecular class A [1] that belongs to both functional groups 2 be and $2 \mathrm{~d}$ of Bush and Jacob classification of 2010 [2]. Majority of studies ( $n=22$, 81.5\%) reported predominance of blaCTX-M gene among isolates, particularly CTX-M-15. Predictors of ESBL-E included increased age, hospital admissions, previous use of antibiotics, and paramedical use of herbs. Conclusion. Few studies have been conducted at a molecular level to understand the genetic basis of increased resistance among members of ESBL-E in ECSA. Limited molecular studies in the ECSA region leave a gap in estimating the burden and risk posed by the carriage of ESBL genes in these countries. We found a high prevalence of ESBL-E most carrying CTX-M enzyme in ECSA with a greater variation between countries. This could be an important call for combined (regional or global) efforts to combat the problem of antimicrobial resistance (AMR) in the region. Antibiotic use and hospital admission increased the carriage of ESBL-E, while poor people contributed little to the increase of AMR due to lack of access and failure to meet the cost of healthcare compared to high income individuals.
\end{abstract}




\section{Introduction}

Pathogenic bacteria evolve to resist the actions of antimicrobials through acquired and intrinsic mechanisms including production of $\beta$-lactamase enzymes, which inactivates antibiotic and decreases its therapeutic value $[1,2]$. Extended-spectrum $\beta$-lactamases produced by many gram-negative bacteria, mostly Enterobacteriaceae, are able to hydrolyze penicillins, cephalosporin, and monobactams . They are mostly effective against a range of $\beta$-lactam drugs including ceftazidime, ceftriaxone, cefotaxime, and aztreonam [3-5]. In many cases, resistance to these antibiotics is transferred among bacteria through gene transfer systems of mobile genetic elements carried in bacteria plasmids or transposons by bacterial recombination process that involves conjugation, transformation, and transduction.

The world prevalence of community and hospital-acquired ESBL-E is increasing tremendously. Bacteria harboring ESBL enzymes are currently the number one critical pathogens posing a major threat to human health [6]. The spread and dissemination of infections caused by ESBL-E are associated with increased morbidity and mortality, health care costs, the need for development of new wide-spectrum antimicrobials and lengthy hospital stay of infected patients. This is because of a major decrease in therapeutic value of mostly used drugs as a result of resistance [7-11].

There has been a significant advancement in the understanding of ESBL-producing bacteria epidemic which was previously related to hospital-acquired infections $[12,13]$. Recent increased recovery of ESBL-E from community and environmental samples [14-16], especially E. coli commonly causing community acquired urinary tract infections (UTIs) [17], indicates a probability of the shift of importation of ESBL-producing bacteria to hospitals rather than vice versa. The spread of community acquired ESBL carrying pathogens is accelerated by between-persons transmission of ESBL bacteria in the communities. Some studies suggest there are significantly higher transmission rates of ESBL-producing bacteria among community households as compared to hospital transmissions [18]. However, detailed studies describing the ESBL-E reservoirs and transmission routes in diverse settings are still limited.

In most poor resource countries of East, Central, and Southern Africa, there is lack of routine surveillance systems that could estimate the magnitude and risk factors as well as clinical outcomes associated with ESBL Enterobacteriaceae [19]. The overuse and misuse of antimicrobial agents in the environmental sector, agriculture, and human and veterinary medicine propel the spread of antimicrobial resistance among infectious bacteria. Other factors such as easy access to antibiotics, weak health systems, environmental contaminations, poor hygiene and sanitation services or practices, incomplete decontamination of medical devices, and lack of laboratory capacities for pathogens detection and surveillance have been described as important drives of the increasing resistance among members of ESBL Enterobacteriaceae in the region [20-22].
1.1. Objective. To better understand genetic determinants of resistance among ESBL-producing Enterobacteriaceae in East, Central, and Sothern Africa, we sought to summarize molecular evidence on the prevalence of ESBL-E and associated genes at community and hospital settings.

\section{Material and Methods}

This review and meta-analysis of prevalence has been conducted in compliance with Preferred Reporting Items for Systematic Reviews and Meta-Analyses (PRISMA) statement and checklist [23].

2.1. The Study Area. The review provides information on published articles from East, Central, and Southern Africa (ECSA) countries in accordance with Africa Union Countries profile [24]. We included six countries from the eastern Africa which are Tanzania, Kenya, Uganda, Rwanda, Burundi, and Ethiopia; five countries from central Africa, Republic of Chad, Central African Republic, Democratic Republic of Congo, Republic of the Congo, and Gabon; and Seven southern African countries of Botswana, Angola, South Africa, Malawi, Namibia, Zambia, and Zimbabwe.

2.2. Literature Search. Literature search of peer reviewed studies was conducted on PubMed and Google Scholar databases for the available molecular studies on ESBL-E in hospitals and community settings in ECSA. The search strategy included the following index terms and Boolean operators (extended-spectrum beta-lactamase OR extendedspectrum OR beta-lactamase OR ESBL beta-lactamases OR beta-lactamase OR Enterobacteriaceae) AND Community OR Hospital AND (Botswana OR Burundi OR Central African Republic OR Chad OR Congo OR Democratic Republic Congo OR "Democratic Republic of the Congo" OR Zaire OR Ethiopia OR Gabon OR Kenya OR Malawi OR Rwanda OR South Africa OR "South Africa" OR Tanzania OR Uganda OR Zambia OR Zimbabwe OR Southern Africa OR Eastern Africa OR East Africa OR Central Africa).

2.3. Study Inclusion and Exclusion Criteria. All available published molecular studies (involving genotypic characterization of ESBL) in English language on human subject reporting ESBL-E in hospitals and communities in ECSA were considered eligible. Unpublished studies, editorials, letters, studies on nonhuman subjects, studies published in other languages than English, and studies that did not utilize molecular tools were excluded. All studies available on the selected databases at the time of data extraction were examined (inception to November 2019).

2.4. Data Extraction. Data extraction checklist was developed to guide the acquisition of the information which included name of the author(s), year of publication, study setting (community or hospital), study design, subjects/ target population, source of isolates/specimen, clinical 
samples, sample size, bacteriological methods for estimating ESBL's, molecular methods used, isolate species, number of isolates analysed, ESBL positive isolates, genes encoding for ESBL identified and risk factors associated with ESBL infection if studied.

2.5. Data Analysis. Acquired data were entered into Excel spreadsheet and statistical analysis was done using Stata version 12 (STATA Corporation, College Station, TX, USA). We performed a random effect meta-analysis to determine heterogeneity of ESBL-E prevalence in ECSA. Decision to perform random effect meta-analysis over fixed effect metaanalysis was made due to an assumption that the difference in ESBL-E prevalence among studies in ECSA is attributed to different factors such as study settings (hospital and community) and different laboratory methods used for detection of ESBL-E. A new program in STATA (Metanprop) specific for pooling binomial data including methods of computation of the $95 \%$ confidence intervals (CI), continuity correct, and the Freeman-Tukey transformation was used [25]. The risk of publication bias was assessed using funnel plot and Begg's rank correlation test for funnel plot asymmetry [26].

\section{Results}

A total of 27 studies involving molecular characterization of ESBL-E that were retrieved from 11 countries in Eastern, Central, and Southern Africa region met the inclusion criteria and were included in this review (Figure 1). The publication year of the studies ranged between 2005 and 2019. Cross-sectional studies comprised $93 \%$ of all studies [9, 27-41]. Majority of studies (74\%) were conducted in hospital settings, $15 \%$ in the community settings, and others in both hospital and community settings.

\subsection{Bacteriological Methods for Estimating ESBL Producers.} The most utilized method of ESBL estimation and confirmation was double disk synergy test (DDST) (59\%); one study used both DDST and combination disk method (CD) [42]. An automated VITEX 2 systems was used in $22 \%$ of the studies $[32,35,39,40,43,44]$ while $15 \%$ used E-test $[9,41,45,46]$.

3.2. Molecular Tools Used for the Detection of ESBL Genes. Three molecular tools were used in all 27 studies to detect the presence of ESBL encoding genes among isolates. These included microarray, polymerase chain reaction (PCR), and sequencing. Seventeen studies $(63 \%)$ used both PCR and sequencing, other studies either utilized PCR (22\%) $[9,30,33,41,46-48]$ or sequencing $(11 \%)[22,29,49]$, and one study used microarray alone [32].

3.3. Country Prevalence of ESBL-Producing Enterobacteriaceae from Human in Eastern, Central, and Southern Africa. A total of 20,225 ESBL-producing isolates from various clinical samples were reported in 27 studies across Eastern, Central, and Southern Africa. Eighty-one percent of the studies isolated both Klebsiella pneumoniae (K. pneumoniae) and Escherichia coli (E. coli). Of the studies, $63 \%$ reported predominance of $E$. coli among isolates, Klebsiella pneumoniae was dominant in $33 \%$, and $5 \%$ of the studies reported that Proteus spp. was dominant. Other Enterobacteriaceae isolated in a small proportion that could not dominate were Enterobacter spp., Citrobacter spp., Morganella spp., and Providencia spp. Prevalence of ESBL-E varied across the countries in ECSA; the highest prevalence was observed in DR Congo followed by Tanzania and Malawi at the rate of $92 \%(95 \% \mathrm{CI}=90-94 \%)$ [29], $89 \%$ (95\%, CI $=82-95 \%),[50]$ and $62 \%(95 \%, C I=61-62 \%)$ [51], respectively. The lowest individual study prevalence was reported in Zimbabwe [34] (Figure 2).

\subsection{Pooled Prevalence of ESBL-Producing Enterobacteriaceae} in East, Central, and Southern Africa. A random pooled prevalence of ESBL-producing Enterobacteriaceae in human from 11 ECSA countries with available data was 38\% (95\% $\mathrm{CI}=24-53 \%)$. The pooled prevalence of ESBL-E in hospital settings was $41 \%(95 \% \quad \mathrm{CI}=23-58 \%)$ and $34 \%(95 \%$ $\mathrm{CI}=8-60 \%)$ in community settings. The variation in the prevalence of ESBL-E attributable to heterogeneity in ECSA was very high $\left(\mathrm{I}^{2}=99.89 \% ; p \leq 0.001\right)$ (Figure 2$)$.

3.5. Epidemiology of Extended-Spectrum $\beta$-Lactamases in ECSA. Genes encoding ESBL enzymes detected from clinical isolates with ESBL-E phenotypes in ECSA were those of Ambler molecular class A [1] that belongs to both functional groups 2be and 2d of Bush and Jacob classification (2010) [2]. These included SHV, CTX-M \& TEM ( $\beta$-lactamases of subgroups $2 \mathrm{~b}, 2 \mathrm{be}$, and $2 \mathrm{ber}$ ), and OXA like $\beta$-lactamases of subgroup $2 \mathrm{~d}$ (Table 1 ).

Majority of studies $(n=22,82 \%)$ reported predominance of blaCTX-M genes among isolates particularly CTX-M-15, predominance of SHV gene was reported in three studies $(n=3,11 \%)[9,37,45]$, and $7 \%$ reported equal proportion of TEM and CTX-M genes detected [49]. The detection of CTX-M genes was mostly on isolates from stool, rectal swabs, and wound swabs; other extended-spectrum $\beta$-lactamase coding genes including SHV, TEM, and OXA type were mostly detected in $K$. pneumoniae commonly recovered from blood samples [45, 46, 48, 49].

3.6. Factors Associated with ESBL-E Infections in ECSA. Five of 27 articles reviewed stressed out factors associated with ESBL-E infections to include increased age, hospital admissions, and previous use of antibiotic. A study in Gabon found that hospitalization and at least 5 years of age were the risks for the carriage of ESBL Enterobacteriaceae in children [43]. Higher ESBL-E carriage was strongly associated with treatment of HIV infection in Zimbabwean children [34]. In an area where antibiotics could not be afforded due to poverty and civil war, ESBL-E carriage in children was associated with high income families [52]. Increased number of children in the household, high median age, previous use 


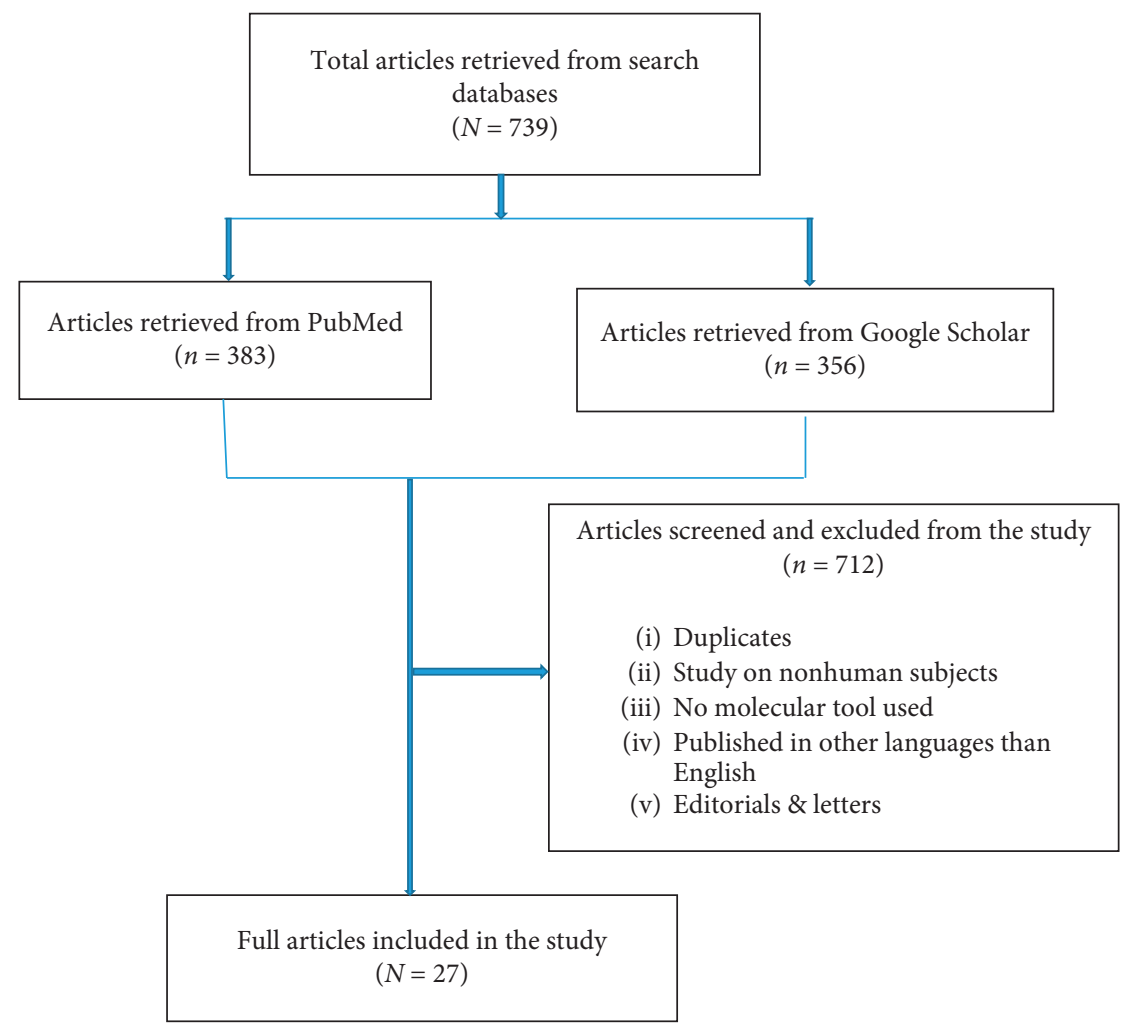

Figure 1: The flow of study selection process.

of antibiotics, and use of local herbs for paramedical purposes were also predictors of ESBL-E carriage [36-40].

\section{Discussion}

In the present review, we present the summary of molecular determinants and the pooled prevalence of ESBL-producing Enterobacteriaceae in Eastern, Central, and Southern Africa. Few studies have been conducted to determine ESBL genes in these countries. Tanzania did most of the genotypic characterization of ESBLs while in other ECSA countries there was no any molecular work on AMR due to ESBL production by members of Enterobacteriaceae. The overall pooled prevalence was high (38\%), close to previous findings by Sonda et al., [54] but higher than that found by Tansarli et al. [19] and Bulabula et al. [55].

According to the data generated from the present review, there was low pooled prevalence of ESBL-E in Zimbabwe and higher in DR Congo, Tanzania, and Malawi. Comparing our study with previous findings, it is surprising to find the prevalence of ESBL-producing pathogens in Malawi has increased by $89 \%$ within 12 years since the introduction of third-generation cephalosporins. The rate of resistance due to ESBL production at the time of cephalosporins introduction in Malawi in 2005 was very low (0.07\%); the reason for low prevalence at that time was tight restriction on the use of antibiotics [45]. However, the rate rose rapidly to $92 \%$ in 2017 indicating either misuse of third-cephalosporin drugs or loosened restrictions on the antibiotic use [51].
The prevalence of ESBL-E was higher in the hospital settings than the community settings; this was similar to finding of others $[19,56]$. We noted that majority of isolates were obtained from cultures of patients with nosocomial infections leading to the higher rate of ESBL genes detection in hospital settings. Similarly, it has been described elsewhere that most nosocomial infections are associated with ESBL-producing bacteria [9, 22, 37, 43, 49, 57]. Our findings complement previous review by Tansarli et al. [19]. These findings can suggest possible influx of ESBL pathogens into the communities from the hospitals. However, bringing together various disciplines to collectively investigate the burden of ESBL pathogens in the environment, human, and animals interface could provide information on the contribution of these different reservoirs to the ESBL-E epidemic.

Higher prevalence of ESBL-E in ECSA according to this review could be attributed to some factors including the focus of the studies reviewed and resistant genes targeted. Many studies across these countries reporting ESBL-E from human either were focused on certain ESBL bacteria, mainly $E$. coli and $K$. pneumoniae [22, 42, 49], or targeted the detection of specific ESBL genes [32, 35, 38, 40, 41]. Therefore, there were high chances of escalating the prevalence of ESBL producers without inclusion of other $\beta$-lactamases in the studies. However, there could also be inadequate infection control in healthcare systems contributing to the increased spread of ESBL-E in these poor resource countries as previously suggested [58]. Selective pressure due to heavy use of $\beta$-lactam antibiotics as a first- 


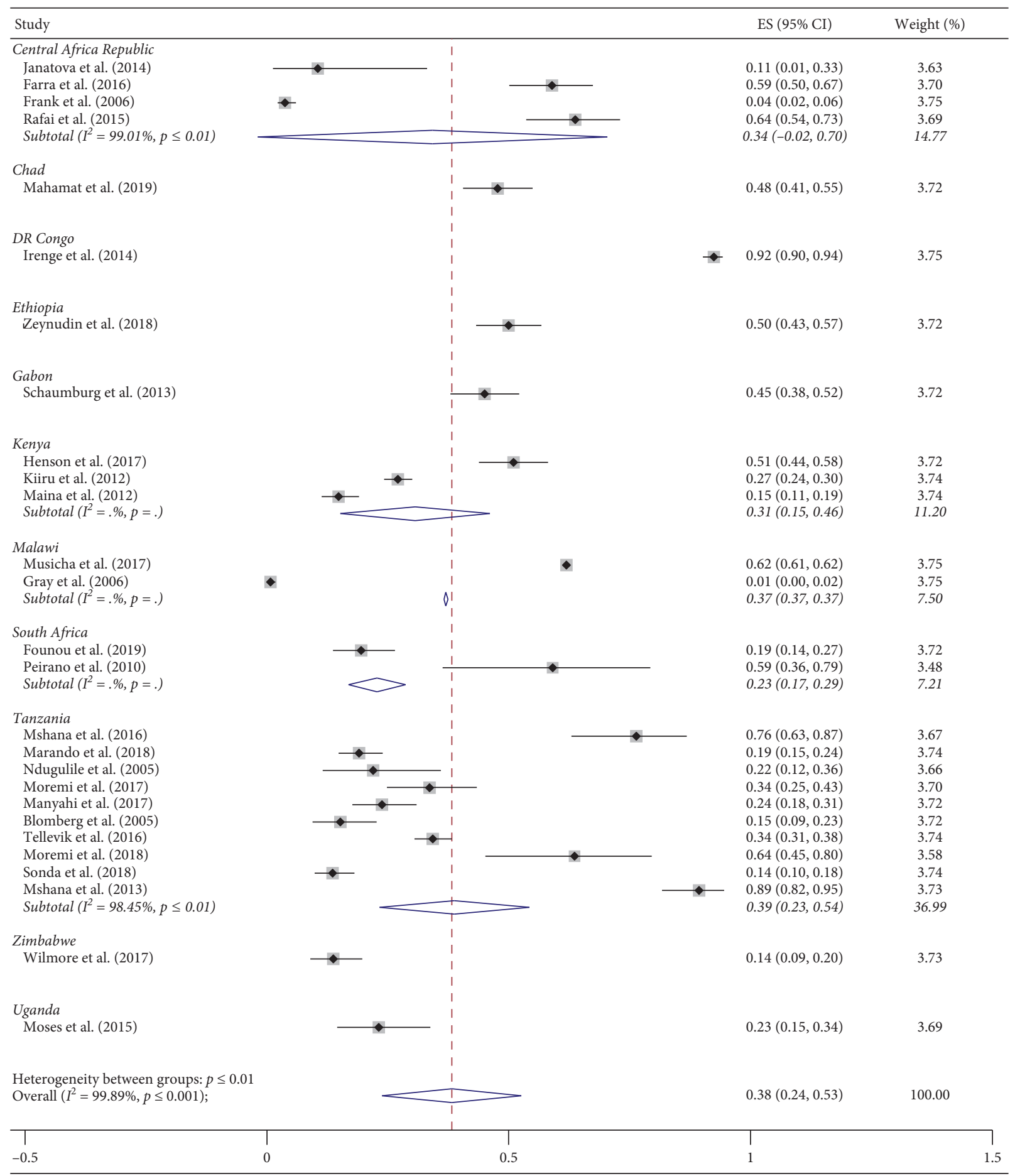

FIGURE 2: Individual study prevalence and pooled prevalence of ESBL-producing Enterobacteriaceae in East, Central, and Southern Africa (the red line indicates statistical significance of the results).

line treatment of infections caused by Enterobacteriaceae has been described to increase the spread of resistance and hence could lead to high prevalence in ECSA [32, 42]. Increased carriage of ESBL-E has also been associated with the use of local herbs such as Aloe vera and other herbal extracts used for paramedical purposes [35]. In most instances, poverty has been linked with increased incidence of ESBL-E. However, our study suggests that people who are poor contribute little to the increased spread of resistance among member of ESBL-E compared to those who can afford to buy and use antibiotics. Since majority of the studies were from hospital settings, it could be that the poor are not well represented due to either lack of access or failure to meet the cost of healthcare.

The fact that few molecular studies have been conducted in the ECSA region undermines the understanding of ESBL 
TABle 1: Prevalence of ESBL-producing Enterobacteriaceae from human in Eastern, Central, and Southern Africa.

\begin{tabular}{|c|c|c|c|c|c|}
\hline $\begin{array}{l}\text { Number of } \\
\text { article (s) }\end{array}$ & Country & Settings & $\begin{array}{c}\text { Prevalence } \\
(\%)\end{array}$ & ESBL gene(s) & Reference \\
\hline \multirow{3}{*}{3} & \multirow{3}{*}{$\begin{array}{c}\text { Central Africa } \\
\text { Republic }\end{array}$} & Hospital & 59 & blaCTX-M-15 & {$[52]$} \\
\hline & & \multirow{2}{*}{ Community } & 11 & $\begin{array}{c}\text { blaCTX-M-15, blaTEM-1, blaOXA-1, blaSHV-2a, \& } \\
\text { blaSHV-62 }\end{array}$ & {$[33]$} \\
\hline & & & 4 & blaCTX-M & {$[31]$} \\
\hline 1 & Chad & Hospital & 48 & $\begin{array}{c}\text { blaCTX-M-9, blaCTX-M-14, blaCTX-M-15, blaCTX-M- } \\
\text { 27, blaTEM-1, \& blaOXA-1 }\end{array}$ & {$[30]$} \\
\hline 1 & DR Congo & Hospital & 92 & blaCTX-M-1 & {$[29]$} \\
\hline 1 & Ethiopia & Hospital & 50 & blaCTX-M-15, blaSHV, \& blaTEM & {$[32]$} \\
\hline 1 & Gabon & Hospital & 45 & blaCTX-M-15, blaCTX-M-8, blaCTX-M-1, \& blaTEM & [43] \\
\hline \multirow{3}{*}{3} & \multirow{3}{*}{ Kenya } & Hospital & 51 & $\begin{array}{c}\text { blaCTX-M-15, blaSHV-2, blaSHV-12, blaSHV-27, } \\
\text { blaOXA-1, \& blaOXA-10 }\end{array}$ & {$[22]$} \\
\hline & & Community & 15 & $\begin{array}{c}\text { blaSHV, blaCTX-M, \& blaTEM } \\
\text { blaSHV-5, blaSHV-12, blaSHV-52, blaCTX-M-8, blaCTX- }\end{array}$ & {$[46]$} \\
\hline & & $\begin{array}{l}\text { Hospital \& } \\
\text { community }\end{array}$ & 27 & $\begin{array}{c}\text { M-14, blaCTX-M-9, blaCTX-M-15, blaCTX-M-3, blaCTX- } \\
\text { M-1, blaTEM-12, bla TEM-125, blaTEM-50, blaTEM-78, } \\
\text { blaTEM-109, blaTEM-152, \& blaTEM-158 }\end{array}$ & {$[42]$} \\
\hline \multirow[b]{2}{*}{2} & \multirow[b]{2}{*}{ Malawi } & \multirow[b]{2}{*}{ Hospital } & 62 & blaCTX-M & {$[51]$} \\
\hline & & & 1 & $\begin{array}{c}\text { blaSHV-11, blaSHV-12, blaSHV-27, blaCTX-M-15, \& } \\
\text { blaTEM-63 }\end{array}$ & {$[45]$} \\
\hline \multirow{3}{*}{2} & \multirow{2}{*}{ South Africa } & \multirow{3}{*}{ Hospital } & 19 & blaCTX-M-15, blaTEM-1b, blaSHV-1, \& blaOXA-1 & {$[49]$} \\
\hline & & & 59 & blaCTX-M-3, blaCTX-M-14, blaCTX-M-15, \& blaSHV-2 & {$[44]$} \\
\hline & \multirow{10}{*}{ Tanzania } & & 76 & blaCTX-M-15 & {$[40]$} \\
\hline \multirow{9}{*}{10} & & Community & 34 & $\begin{array}{c}\text { blaCTX-M-9, blaCTX-M-15, blaCTX-M-55, blaTEM-1, } \\
\text { blaSHV-1, \& blaSHV-11 }\end{array}$ & {$[35]$} \\
\hline & & \multirow{3}{*}{$\begin{array}{l}\text { Hospital \& } \\
\text { community }\end{array}$} & 24 & blaCTX-M-15 \& blaSHV-12 & {$[41]$} \\
\hline & & & 34 & blaCTX-M-14, blaCTX-M-15, blaSHV-5, \& blaSHV-12 & [53] \\
\hline & & & 64 & blaCTX-M-15 & [39] \\
\hline & & \multirow{5}{*}{ Hospital } & 14 & blaCTX-M & {$[38]$} \\
\hline & & & 89 & $\begin{array}{c}\text { blaCTX-M-15, blaSHV-11, blaTEM-1, blaTEM-104, \& } \\
\text { blaTEM-176 }\end{array}$ & {$[50]$} \\
\hline & & & 19 & $\begin{array}{c}\text { blaSHV-1, blaSHV-11, blaSHV-27, blaSHV-33, blaCTX- } \\
\text { M-15, blaTEM-1B, \& blaOXA-1 }\end{array}$ & {$[36]$} \\
\hline & & & 22 & blaSHV-12, blaSHV-28, blaCTX-M-15, \& blaTEM-1 & [37] \\
\hline & & & 15 & blaSHV-2a, blaSHV-12, blaCTX-M-15, \& blaTEM-63 & [9] \\
\hline 1 & Uganda & Hospital & 23.2 & blaSHV, blaCTX-M, \& blaTEM & [27] \\
\hline 1 & Zimbabwe & Hospital & 14 & $\begin{array}{c}\text { blaCTX-M-3, blaCTX-M-14, blaCTX-M-15, blaCTX-M- } \\
\text { 27, blaTEM, \& blaSHV }\end{array}$ & {$[34]$} \\
\hline
\end{tabular}

gene diversity implicated in hospital and community acquired infections caused by ESBL-E strains. Most studies done with regard to ESBL-E in the region looked at identifying the pathogens involved and phenotypic resistance patterns rather than the genes responsible for such resistance. Similar to other studies [59,60], we found CTX-M genes particularly CTX-M-15 dominating other types of ESBL genes in both hospital and community isolates. With few allelic groups of ESBLs reported in the reviewed studies, our findings indicated that CTX-M-15 is more common than other ESBL types found in both clinical and nonclinical samples $[35,38,40,41,60-63]$. Previous studies have suggested that commensal isolates originating from infection of the gut and intestines carried different ESBL genes depending on bacteria species, plasmids, and location of the chromosome bearing the resistant gene [33]. However, in this study we observed invariability of ESBL gene types between community and hospital isolates across the studies.

The importance of genotypic detection of ESBL genes in bacteria over phenotypic screening cannot be ignored; this is because these genes are carried on plasmids permitting higher chances of horizontal transfer which cannot be detected phenotypically. Phenotypic screening for ESBL pathogens is useful for surveillance purposes but they can underestimate the burden of resistance posed by ESBL pathogens. Previous reports detected more resistant ESBL producers when molecular methods were used compared to phenotypic methods [27]. For definitive therapy selection, relying on phenotypic determination of ESBL enzymes alone may not be sufficient. Therefore, molecular detection is inevitable.

4.1. Strengths and Limitations. One of the strengths of this review is that we included studies published in English which is the official language of many countries in the study area. Searching Google Scholar database provided an advantage to retrieve articles that could have not been published in highly indexed journals. Institutional subscription also allowed retrieval of articles that were not available for 
free access. However, the study was limited by the challenge encountered in determining true prevalence because some studies were laboratory surveillance based and did not provide the actual sample size or number of participants from which the clinical samples were drawn.

4.2. Conclusion and Recommendations. Few studies have been conducted at a molecular level to understand the genetic basis of increased resistance among members of ESBL$\mathrm{E}$ in ECSA. Limited molecular studies in the ECSA region leave a gap in estimating the burden and risk posed by the carriage of ESBL genes in these countries. We found a high prevalence of ESBL-E most carrying CTX-M enzyme in ECSA with a greater variation between countries. This could be an important call for combined (regional or global) efforts to combat the problem of AMR in the region. Antibiotic use and hospital admission increased the carriage of ESBL-E, while poor people contributed little to the increase of AMR due to lack of access and failure to meet the cost of healthcare compared to high income individuals.

\section{Conflicts of Interest}

The authors declare no conflicts of interest.

\section{Acknowledgments}

The authors did not receive any financial support for conducting this review or publication.

\section{References}

[1] R. P. Ambler, A. F. W. Coulson, J. M. Frère et al., "A standard numbering scheme for the class A $\beta$-lactamases," Biochemical Journal, vol. 276, no. 1, pp. 269-270, 1991.

[2] K. Bush and G. A. Jacoby, "Updated functional classification of $\beta$-lactamases," Antimicrobial Agents and Chemotherapy, vol. 54, no. 3, pp. 969-976, 2010.

[3] D. L. Paterson and R. A. Bonomo, "Extended-spectrum $\beta$-lactamases: a clinical update," Clinical Microbiology Reviews, vol. 18, no. 4, pp. 657-686, 2005.

[4] K. Bush, G. A. Jacoby, and A. A. Medeiros, "A functional classification scheme for beta-lactamases and its correlation with molecular structure," Antimicrobial Agents and Chemotherapy, vol. 39, no. 6, pp. 1211-1233, 1995.

[5] P. A. Bradford, "Extended-spectrum $\beta$-lactamases in the 21st century: characterization, epidemiology, and detection of this important resistance threat," Clinical Microbiology Reviews, vol. 14, no. 4, pp. 933-951, 2001.

[6] E. Tacconelli, E. Carrara, A. Savoldi et al., "Discovery, research, and development of new antibiotics: the WHO priority list of antibiotic-resistant bacteria and tuberculosis," The Lancet Infectious Diseases, vol. 18, no. 3, pp. 318-327, 2018.

[7] J. M. Bell, J. D. Turnidge, A. C. Gales, M. A. Pfaller, and R. N. Jones, "Prevalence of extended spectrum $\beta$-lactamase (ESBL)-producing clinical isolates in the Asia-Pacific region and South Africa: regional results from SENTRY Antimicrobial Surveillance Program (1998-99)," Diagnostic Microbiology and Infectious Disease, vol. 42, no. 3, pp. 193-198, 2002 .
[8] S. E. Mshana, M. Matee, and M. Rweyemamu, "Antimicrobial resistance in human and animal pathogens in Zambia, Democratic Republic of Congo, Mozambique and Tanzania: an urgent need of a sustainable surveillance system," Annals of Clinical Microbiology and Antimicrobials, vol. 12, no. 1, p. 28, 2013.

[9] B. Blomberg, R. Jureen, K. P. Manji et al., "High rate of fatal cases of pediatric septicemia caused by gram-negative bacteria with extended-spectrum beta-lactamases in Dar es Salaam, Tanzania," Journal of Clinical Microbiology, vol. 43, no. 2, pp. 745-749, 2005.

[10] R. Ben-Ami, J. Rodríguez-Baño, H. Arslan et al., "A multinational survey of risk factors for infection with extendedspectrum $\beta$-lactamase-producing enterobacteriaceae in nonhospitalized patients," Clinical Infectious Diseases, vol. 49, no. 5, pp. 682-690, 2009.

[11] W. C. Rottier, H. S. M. Ammerlaan, and M. J. M. Bonten, "Effects of confounders and intermediates on the association of bacteraemia caused by extended-spectrum $\beta$-lactamaseproducing Enterobacteriaceae and patient outcome: a metaanalysis," Journal of Antimicrobial Chemotherapy, vol. 67, no. 6, pp. 1311-1320, 2012.

[12] S. S. Magill, J. R. Edwards, W. Bamberg et al., "Multistate point-prevalence survey of health care-associated infections," New England Journal of Medicine, vol. 370, no. 13, pp. 1198-1208, 2014.

[13] R. Podschun and U. Ullmann, "Klebsiella spp. as nosocomial pathogens: epidemiology, taxonomy, typing methods, and pathogenicity factors," Clinical Microbiology Reviews, vol. 11, no. 4, pp. 589-603, 1998.

[14] W. D. Tanner, J. A. VanDerslice, R. K. Goel et al., "Multi-state study of Enterobacteriaceae harboring extended-spectrum beta-lactamase and carbapenemase genes in U.S. drinking water," Scientific Reports, vol. 9, p. 3938, 2019.

[15] A. Haque, A. Yoshizumi, T. Saga, Y. Ishii, and K. Tateda, "ESBL-producing Enterobacteriaceae in environmental water in Dhaka, Bangladesh," Journal of Infection and Chemotherapy, vol. 20, no. 11, pp. 735-737, 2014.

[16] K. Zurfluh, H. Hächler, M. Nüesch-Inderbinen, and R. Stephan, "Characteristics of extended-spectrum $\beta$-lactamase- and carbapenemase-producing enterobacteriaceae isolates from rivers and lakes in Switzerland," Applied and Environmental Microbiology, vol. 79, no. 9, pp. 3021-3026, 2013.

[17] J. D. Pitout and K. B. Laupland, "Extended-spectrum $\beta$-lactamase-producing Enterobacteriaceae: an emerging publichealth concern," The Lancet Infectious Diseases, vol. 8, no. 3, pp. 159-166, 2008.

[18] M. Hilty, B. Y. Betsch, K. Bögli-Stuber et al., "Transmission dynamics of extended-spectrum $\beta$-lactamase-Producing enterobacteriaceae in the tertiary care hospital and the household setting," Clinical Infectious Diseases, vol. 55, no. 7, pp. 967-975, 2012.

[19] G. S. Tansarli, P. Poulikakos, A. Kapaskelis, and M. E. Falagas, "Proportion of extended-spectrum -lactamase (ESBL)-producing isolates among Enterobacteriaceae in Africa: evaluation of the evidence--systematic review," Journal of Antimicrobial Chemotherapy, vol. 69, no. 5, pp. 1177-1184, 2014.

[20] S. Y. Essack, A. T. Desta, R. E. Abotsi, and E. E. Agoba, "Antimicrobial resistance in the WHO African region: current status and roadmap for action," Journal of Public Health, vol. 39, no. 1, p. fdw015, 2016. 
[21] S. Kariuki and G. Dougan, "Antibacterial resistance in subSaharan Africa: an underestimated emergency," Annals of the New York Academy of Sciences, vol. 1323, no. 1, pp. 43-55, 2014.

[22] S. P. Henson, C. J. Boinett, M. J. Ellington et al., "Molecular epidemiology of Klebsiella pneumoniae invasive infections over a decade at Kilifi County Hospital in Kenya," International Journal of Medical Microbiology, vol. 307, no. 7, pp. 422-429, 2017.

[23] D. Moher, A. Liberati, J. Tetzlaff, and D. G. Altman, "Preferred reporting Items for systematic reviews and meta-analyses: the PRISMA statement," PLoS Medicine, vol. 6, no. 7, Article ID e1000097, 2009.

[24] "Member States | African Union," July 2019, https://au.int/en/ member_states/countryprofiles 2 .

[25] V. N. Nyaga, M. Arbyn, and M. Aerts, "Metaprop: a Stata command to perform meta-analysis of binomial data," Archives of Public Health $=$ Archives Belges de Sante Publique, vol. 72, no. 1, pp. 39-10, 2014.

[26] C. B. Begg and M. Mazumdar, "Operating characteristics of a rank correlation test for publication bias," Biometrics, vol. 50, no. 4, p. 1088, 1994.

[27] A. Moses, F. Bwanga, Y. Boum, and J. Bazira, "Prevalence and genotypic characterization of extended- spectrum beta-lactamases produced by gram negative bacilli at a tertiary care hospital in Rural South Western Uganda," British Microbiology Research Journal, vol. 4, no. 12, pp. 1541-1550, 2014.

[28] G. V. Bloemberg, S. Polsfuss, V. Meyer, E. C. Böttger, and M. Hombach, "Evaluation of the AID ESBL line probe assay for rapid detection of extended-spectrum $\beta$-lactamase (ESBL) and KPC carbapenemase genes in Enterobacteriaceae," Journal of Antimicrobial Chemotherapy, vol. 69, no. 1, pp. 85-90, 2014.

[29] L. M. Irenge, L. Kabego, O. Vandenberg, R. B. Chirimwami, and J. L. Gala, "Antimicrobial resistance in urinary isolates from inpatients and outpatients at a tertiary care hospital in South-Kivu Province (Democratic Republic of Congo)," BMC Research Notes, vol. 7, no. 1, pp. 374-376, 2014.

[30] O. O. Mahamat et al., "High prevalence and characterization of extended-spectrum $\beta$-lactamase producing Enterobacteriaceae in Chadian hospitals," BMC Infectious Diseases, vol. 19, no. 1, pp. 1-7, 2019.

[31] T. Frank, G. Arlet, V. Gautier, A. Talarmin, and R. Bercion, "Extended-spectrum $\beta$-Lactamase-producingEnterobacteriaceae, Central African Republic," Emerging Infectious Diseases, vol. 12, no. 5, pp. 863-865, 2006.

[32] A. Zeynudin, M. Pritsch, S. Schubert et al., "Prevalence and antibiotic susceptibility pattern of CTX-M type extendedspectrum $\beta$-lactamases among clinical isolates of gram-negative bacilli in Jimma, Ethiopia," BMC Infectious Diseases, vol. 18, no. 1, pp. 524-610, 2018.

[33] M. Janatova, K. Albrechtova, K. J Petrzelkova et al., "Antimicrobial-resistant enterobacteriaceae from humans and wildlife in Dzanga-Sangha protected area, Central African Republic," Veterinary Microbiology, vol. 171, no. 3-4, pp. 422-431, 2014.

[34] S. M. S. Wilmore, K. Kranzer, A. Williams et al., "Carriage of extended-spectrum beta-lactamase-producing enterobacteriaceae in HIV-infected children in Zimbabwe," Journal of Medical Microbiology, vol. 66, no. 5, pp. 609-615, 2017.

[35] N. Moremi, H. Claus, U. Vogel, and S. E. Mshana, "Faecal carriage of CTX-M extended-spectrum beta-lactamase-producing Enterobacteriaceae among street children dwelling in
Mwanza city, Tanzania," PLoS One, vol. 12, no. 9, Article ID e0184592, 2017.

[36] R. Marando, J. Seni, M. M. Mirambo et al., "Predictors of the extended-spectrum-beta lactamases producing Enterobacteriaceae neonatal sepsis at a tertiary hospital, Tanzania," International Journal of Medical Microbiology, vol. 308, no. 7, pp. 803-811, 2018.

[37] F. Ndugulile, R. Jureen, S. Harthug, W. Urassa, and N. Langeland, "Extended spectrum $\beta$-lactamases among Gram-negative bacteria of nosocomial origin from an Intensive Care Unit of a tertiary health facility in Tanzania," BMC Infectious Diseases, vol. 5, pp. 1-6, 2005.

[38] T. Sonda, H. Kumburu, M. van Zwetselaar et al., "Prevalence and risk factors for CTX-M gram-negative bacteria in hospitalized patients at a tertiary care hospital in Kilimanjaro, Tanzania," European Journal of Clinical Microbiology \& Infectious Diseases, vol. 37, no. 5, pp. 897-906, 2018.

[39] N. Moremi, H. Claus, L. Rutta, M. Frosch, U. Vogel, and S. E. Mshana, "High carriage rate of extended-spectrum betalactamase-producing Enterobacteriaceae among patients admitted for surgery in Tanzanian hospitals with a low rate of endogenous surgical site infections," Journal of Hospital Infection, vol. 100, no. 1, pp. 47-53, 2018.

[40] S. E. Mshana, L. Falgenhauer, M. M. Mirambo et al., "Predictors of blaCTX-M-15 in varieties of Escherichia coli genotypes from humans in community settings in Mwanza, Tanzania," BMC Infectious Diseases, vol. 16, no. 1, pp. 187-189, 2016.

[41] J. Manyahi, S. J Moyo, M. G. Tellevik et al., "Detection of CTX-M-15 beta-lactamases in Enterobacteriaceae causing hospital- and community-acquired urinary tract infections as early as 2004, in Dar es Salaam, Tanzania," BMC Infectious Diseases, vol. 17, no. 1, pp. 282-287, 2017.

[42] J. Kiiru, S. Kariuki, B. M. Goddeeris, and P. Butaye, "Analysis of -lactamase phenotypes and carriage of selected -lactamase genes among Escherichia coli strains obtained from Kenyan patients during an 18-year period," BMC Microbiology, vol. 12, no. 155, pp. 1471-2180, 2012.

[43] F. Schaumburg, A. Alabi, C. Kokou et al., "High burden of extended-spectrum $\quad \beta$-lactamase-producing Enterobacteriaceae in Gabon," Journal of Antimicrobial Chemotherapy, vol. 68, no. 9, pp. 2140-2143, 2013.

[44] G. Peirano, C. H. J. van Greune, and J. D. D. Pitout, "Characteristics of infections caused by extended-spectrum $\beta$-lactamase-producing Escherichia coli from community hospitals in South Africa," Diagnostic Microbiology and Infectious Disease, vol. 69, no. 4, pp. 449-453, 2011.

[45] K. J. Gray, L. K. Wilson, A. Phiri, J. E. Corkill, N. French, and C. A. Hart, "Identification and characterization of ceftriaxone resistance and extended-spectrum $\beta$-lactamases in Malawian bacteraemic enterobacteriaceae," Journal of Antimicrobial Chemotherapy, vol. 57, no. 4, pp. 661-665, 2006.

[46] D. Maina, G. Revathi, S. Kariuki, and H. Ozwara, "Genotypes and cephalosporin susceptibility in extended-spectrum betalactamase producing enterobacteriaceae in the community," The Journal of Infection in Developing Countries, vol. 6, no. 6, pp. 470-477, 2011.

[47] D. Okoche, B. B. Asiimwe, F. A. Katabazi, L. Kato, and C. F. Najjuka, "Prevalence and characterization of carbapenem-resistant enterobacteriaceae isolated from Mulago National Referral Hospital, Uganda," PLoS One, vol. 10, no. 8, Article ID e0135745, 2015.

[48] R. Bercion, D. Mossoro-Kpinde, A. Manirakiza, and A. Le Faou, "Increasing prevalence of antimicrobial resistance 
among Enterobacteriaceae uropathogens in Bangui, Central African Republic," Journal of Infection in Developing Countries, vol. 3, no. 3, pp. 187-190, 2009.

[49] R. C. Founou, L. L. Founou, M. Allam, A. Ismail, and S. Y. Essack, "Whole genome sequencing of extended spectrum $\beta$-lactamase (ESBL)-producing Klebsiella pneumoniae isolated from hospitalized patients in KwaZulu-Natal, South Africa," Scientific Reports, vol. 9, no. 1, pp. 6266-6311, 2019.

[50] S. E. Mshana, T. Hain, E. Domann, E. F. Lyamuya, T. Chakraborty, and C. Imirzalioglu, "Predominance of Klebsiella pneumoniaeST14 carrying CTX-M-15 causing neonatal sepsis in Tanzania," BMC Infectious Diseases, vol. 13, no. 1, p. 466, 2013.

[51] P. Musicha, J. E. Cornick, N. Bar-Zeev et al., "Trends in antimicrobial resistance in bloodstream infection isolates at a large urban hospital in Malawi (1998-2016): a surveillance study," The Lancet Infectious Diseases, vol. 17, no. 10, pp. 1042-1052, 2017.

[52] A. Farra, T. Frank, L. Tondeur et al., "High rate of faecal carriage of extended-spectrum $\beta$-lactamase-producing Enterobacteriaceae in healthy children in Bangui, Central African Republic," Clinical Microbiology and Infection: The Official Publication of the European Society of Clinical Microbiology and Infectious Diseases, vol. 22, no. 10, pp. 891-8e4, 2016.

[53] M. G. Tellevik, B. Blomberg, Ø Kommedal, S. Y. Maselle, N. Langeland, and S. J. Moyo, "High prevalence of faecal carriage of ESBL-producing enterobacteriaceae among children in dar es Salaam, Tanzania," PLoS One, vol. 11, no. 12, Article ID e0168024, 2016.

[54] T. Sonda, H. Kumburu, M. van Zwetselaar et al., "Metaanalysis of proportion estimates of extended-spectrum-betalactamase-producing enterobacteriaceae in East Africa Hospitals," Antimicrobial Resistance and Infection Control, vol. 5, no. 18, pp. 18-19, 2016.

[55] A. N. H. Bulabula, A. Dramowski, and S. Mehtar, "Maternal colonization or infection with extended-spectrum beta-lactamase-producing Enterobacteriaceae in Africa: a systematic review and meta-analysis," International Journal of Infectious Diseases, vol. 64, no. 2017, pp. 58-66, 2017.

[56] S. A. Sangare, A. I. Maiga, I. Guindo et al., "Prevalence of extended-spectrum beta-lactamase-producing Enterobacteriaceae isolated from blood cultures in Africa," Médecine et Maladies Infectieuses, vol. 45, no. 9, pp. 374-382, 2015.

[57] S. Tokajian, J. A. Eisen, G. Jospin, A. Farra, and D. A. Coil, "Whole genome sequencing of extended-spectrum $\hat{I}^{2}$-lactamase producing Klebsiella pneumoniae isolated from a patient in Lebanon," Frontiers in Cellular and Infection Microbiology, vol. 5, p. 32, 2015.

[58] S. L. Knobler, S. M. Lemon, M. Najafi, and T. Burroughs, "Factors contributing to the emergence of resistance," in The Resistance Phenomenon in Microbes and Infectious Disease Vectors: Implications for Human Health and Strategies for Containment: Workshop Summaryp. 336, National Academies Press (US), Washington, DC, USA, 2003.

[59] S. Karanika, T. Karantanos, M. Arvanitis, C. Grigoras, and E. Mylonakis, "Fecal colonization with extended-spectrum beta-lactamase-ProducingEnterobacteriaceaeand risk factors among healthy individuals: a systematic review and metaanalysis," Clinical Infectious Diseases, vol. 63, no. 3, pp. 310-318, 2016.

[60] A. Ibrahimagić, B. Bedenić, F. Kamberović, and S. Uzunović, "High prevalence of CTX-M-15 and first report of CTX-M-3,
CTX-M-22, CTX-M-28 and plasmid-mediated AmpC betalactamase producing Enterobacteriaceae causing urinary tract infections in Bosnia and Herzegovina in hospital and community settings," Journal of Infection and Chemotherapy, vol. 74, no. 3, p. 6, 2015.

[61] P. Espinal, U. Garza-Ramos, F. Reyna et al., "Identification of SHV-type and CTX-M-12 extended-spectrum $\beta$-lactamases (ESBLs) in MultiresistantEnterobacteriaceaefrom Colombian caribbean hospitals," Journal of Chemotherapy, vol. 22, no. 3, pp. 160-164, 2010.

[62] M. Edelstein, M. Pimkin, I. Palagin, I. Edelstein, and L. Stratchounski, "Prevalence and molecular epidemiology of CTX-M extended-spectrum $\beta$-lactamase-producing Escherichia coli and Klebsiella pneumoniae in Russian hospitals," Antimicrobial Agents and Chemotherapy, vol. 47, no. 12, pp. 3724-3732, 2003.

[63] O. B. Ahmed, A. O. Omar, A. H. Asghar, and M. M. Elhassan, "Prevalence of TEM, SHV and CTX-M genes in Escherichia coli and Klebsiella spp urinary isolates from Sudan with confirmed ESBL phenotype," Life Science Journal, vol. 10, no. 2, pp. 191-195, 2013. 